\title{
Predicting Energy Expenditure During Gradient Walking With a Foot Monitoring Device: Model-Based Approach
}

Soon Ho Kim¹, BA; Jong Won Kim², PhD; Jung-Jun Park ${ }^{3}$, PhD; Myung Jun Shin ${ }^{4}$, MD, PhD; MooYoung Choi ${ }^{1}$, $\mathrm{PhD}$

${ }^{1}$ Department of Physics and Astronomy and Center for Theoretical Physics, Seoul National University, Seoul, Republic of Korea

${ }^{2}$ Department of Healthcare Information Technology, Inje University, Gimhae, Republic of Korea

${ }^{3}$ Division of Sport Science, Pusan National University, Busan, Republic of Korea

${ }^{4}$ Department of Rehabilitation Medicine, Pusan National University Hospital, Busan, Republic of Korea

\section{Corresponding Author:}

Jung-Jun Park, PhD

Division of Sport Science

Pusan National University

2, Busandaehak-ro 63beon-gil, Geumjeong-gu

Busan, 46241

Republic of Korea

Phone: 821032851626

Email: jiparkpnu@pusan.ac.kr

\section{Abstract}

Background: Many recent commercial devices aim at providing a practical way to measure energy expenditure. However, those devices are limited in accuracy.

Objective: This study aimed to build a model of energy consumption during walking applicable to a range of sloped surfaces, used in conjunction with a simple, wearable device.

Methods: We constructed a model of energy consumption during gradient walking by using arguments based in mechanics. We built a foot monitoring system that used pressure sensors on the foot insoles. We did experiments in which participants walked on a treadmill wearing the foot monitoring system, and indirect calorimetry was used for validation. We found the parameters of the model by fitting to the data.

Results: When walking at $1.5 \mathrm{~m} / \mathrm{s}$, we found that the model predicted a calorie consumption rate of $5.54 \mathrm{kcal} / \mathrm{min}$ for a woman with average height and weight and $6.89 \mathrm{kcal} / \mathrm{min}$ for an average man. With the obtained parameters, the model predicted the data with a root-mean-square deviation of $0.96 \mathrm{kcal} / \mathrm{min}$ and median percent error of $12.4 \%$.

Conclusions: Our model was found to be an accurate predictor of energy consumption when walking on a range of slopes. The model uses few variables; thus, it can be used in conjunction with a convenient wearable device.

(JMIR Mhealth Uhealth 2019;7(10):e12335) doi: 10.2196/12335

\section{KEYWORDS}

energy expenditure; physical activity; human walking; wearable devices; mHealth

\section{Introduction}

Physical inactivity, despite its well-known health risks [1,2], continues to be a serious public health issue [3]. Recently, various wearable devices, including wristbands and mobile phones, have offered a way to track physical activity throughout the day. Such devices can be used in ambulatory conditions by individuals or in clinical settings to monitor patients' physical activity.
Many of these devices use an accelerometer-based method to predict energy expenditure [4-6]. However, these methods are limited in precision [7]. A basic, common assumption used is that the calorie consumption rate is proportional to the walking velocity. A GPS tracker can then be used to measure the walking distance and then compute the total energy consumption. However, this method is limited in accuracy and may not be feasible indoors. 


\section{Literature Review}

The energetics of human locomotion has been closely studied for decades. Early studies focused on energy expenditure during walking [8-12] and running [13-16], and made comparisons with the energy expenditures of other animals [17]. Most relevantly, studies on walking energetics found a proportional relationship between energy expenditure and the square of the velocity. These early studies showed that reasonable accuracy can be attained with simple relations, despite the complexity of the act of walking. More recently, detailed models of walking dynamics have been presented that examine more closely the mechanics of walking [18-24]. These biomechanical models aim to explain human gait patterns via energy minimization. Also studied have been movements of the arm [25,26] and the head and trunk [27], as well as gait patterns in special groups of interest $[28,29]$. Such models have also been used in the field of robotics in developing walking robots [30].

Previous studies were primarily of academic interest, although inexpensive commercial devices have recently been made available for personal or clinical use. Such devices offer noninvasive ways to measure daily caloric consumption, and they have been assessed by numerous validation studies in the literature [31-36]. The most common types of commercially available products include the wrist-worn accelerometer and devices based on heart rate monitors. Although these devices are good predictors of the number of steps and heart rate, accurate prediction of energy expenditure is yet to be achieved [37]. These validation studies test for various settings; however, they usually lack a discussion of the model or algorithm used in their predictions.

This study proposes a model of walking energetics applicable to a range of slopes. The model is based on a simple equation and uses data from a wearable device. The method uses a foot monitoring system that can sense footsteps, which allows for direct measurement of step frequency. We found that a high-accuracy model can be developed for a range of upward and downward slopes. The fact that it is based on a direct measurement of footsteps allows the device to be versatile and applicable to diverse walking situations. The ability to track expenditure while walking on sloped surfaces is helpful for sloped outdoor ground and also indoor use of stairs or sloped treadmills.

\section{Methods}

\section{Experimental Procedure}

For model development and validation, an experiment was devised in which 73 healthy participants (34 female, 39 male) walked on a treadmill. The participants had a mean age of 43.6 (SD 15.0) years, mean height of 168.3 (SD 10.5) cm, and mean weight of 68.1 (SD 12.1) kg. Participants were selected from healthy volunteers (age 20 to 60 years) who registered in the department of Sport Science, Pusan National University, Busan, Korea. We excluded participants who had cardiovascular, musculoskeletal, or neurological disorders to avoid any confounding factors or biases. The participants were asked to walk on a treadmill at various values of the incline angle, Theta, and speed, $v$. Specifically, the angle was taken to be $0^{\circ}$ (indicating no incline); $4^{\circ}, 9^{\circ}$, and $14^{\circ}$ (uphill); and $-4^{\circ},-9^{\circ}$, and $-14^{\circ}$ (downhill). It was observed that calorie consumption took approximately 30 seconds to stabilize to a linear rate while walking. Each walking measurement lasted approximately 5 minutes to ensure a sufficiently long sample.

Calorie consumption was measured with a COSMED K4b2 portable gas analyzer system. This indirect calorimetry, based on the gas analyzer system, measures oxygen consumption, from which energy expenditure is computed. This method has been validated as an accurate measure through numerous comparative studies [38-40] and is used as a criterion measure in many validation studies [31-33,35-37]. The gas analyzer was worn during the treadmill experiment, and it recorded a time series of cumulative calorie consumption. To eliminate noise associated with the beginning and end of the experiment, we discarded data for the first 50 seconds and the final 10 seconds before computing the energy consumption rate. Then the basal metabolic rate [41] was subtracted to obtain the energy expenditure associated with walking, which is denoted by $P$.

Each participant also wore a foot monitoring system, consisting of shoe insoles equipped with eight pressure sensors. The insole used was a prototype developed by $3 \mathrm{~L}$ Labs (Seoul, Korea), and provided to us for research purposes. A depiction of the foot monitoring system and the experimental setup is given in Figure 1. A Fitbit Surge, a wrist-worn accelerometer device, was also worn by each participant to compare the accuracy of its caloric consumption prediction. This study was approved by the Institutional Review Board of Pusan National University, Busan, Korea. All participants provided written informed consent (PNU IRB/2015_33_HR).

A value of 0,1 , or 2 indicated the pressure on each of the pressure sensors and was recorded with a frequency of $10 \mathrm{~Hz}$, which resulted in an array of 16 integers for each time step of $0.1 \mathrm{~s}$. A snippet from example data is shown in Figure 2. From the pressure sensor data, we were able to extract the step frequency, $f$. We performed this by examining the sum of the pressure sensor values at each time step. An example is shown in Figure 3. Although it is natural to consider the foot to be off the ground when this sum is 0 , this can result in erroneous results if one or more of the pressure sensors remain at a value above 0 throughout the entire step cycle, either due to a faulty sensor or residual pressure. We found that better accuracy was achieved when high and low thresholds were used. This was done by first assigning the on-ground status to the first-time step, and then sequentially assigning either the on-ground or off-ground status to each following time step. If the previous time step was on-ground and the pressure sum was below the lower threshold, we assigned the off-ground status to that time step; if the threshold was not crossed, the time step was left in on-ground status. If the previous status was off-ground, the on-ground status was assigned if the pressure sum was above the upper threshold, and the off-ground status was assigned otherwise. Threshold values between 1 and 10 were tested and compared with manually assigned steps. Lower and upper threshold values of 2 and 5, shown in Figure 3, were found to produce accurate results. 
Figure 1. Illustration of the foot monitoring system (left) and a picture of a participant walking on an uphill treadmill wearing the K4b2 portable gas analyzer (right).
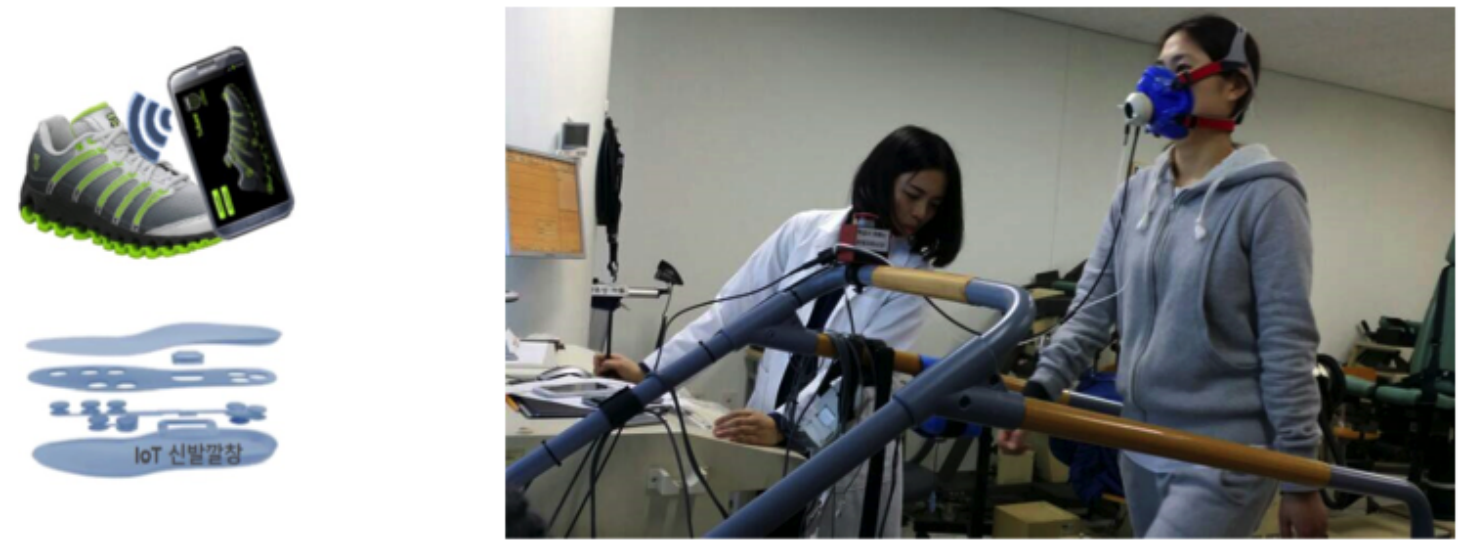

Figure 2. A sample of 4 seconds of raw data from the pressure sensors. The vertical position of each number of the array indicates the time, ordered from top to bottom at an increment of 0.1 seconds. Each column denotes a sensor, with left foot and right foot separated. The colored portions indicate when our algorithm decided the foot was off the ground.

\section{Left Sensors Right Sensors}

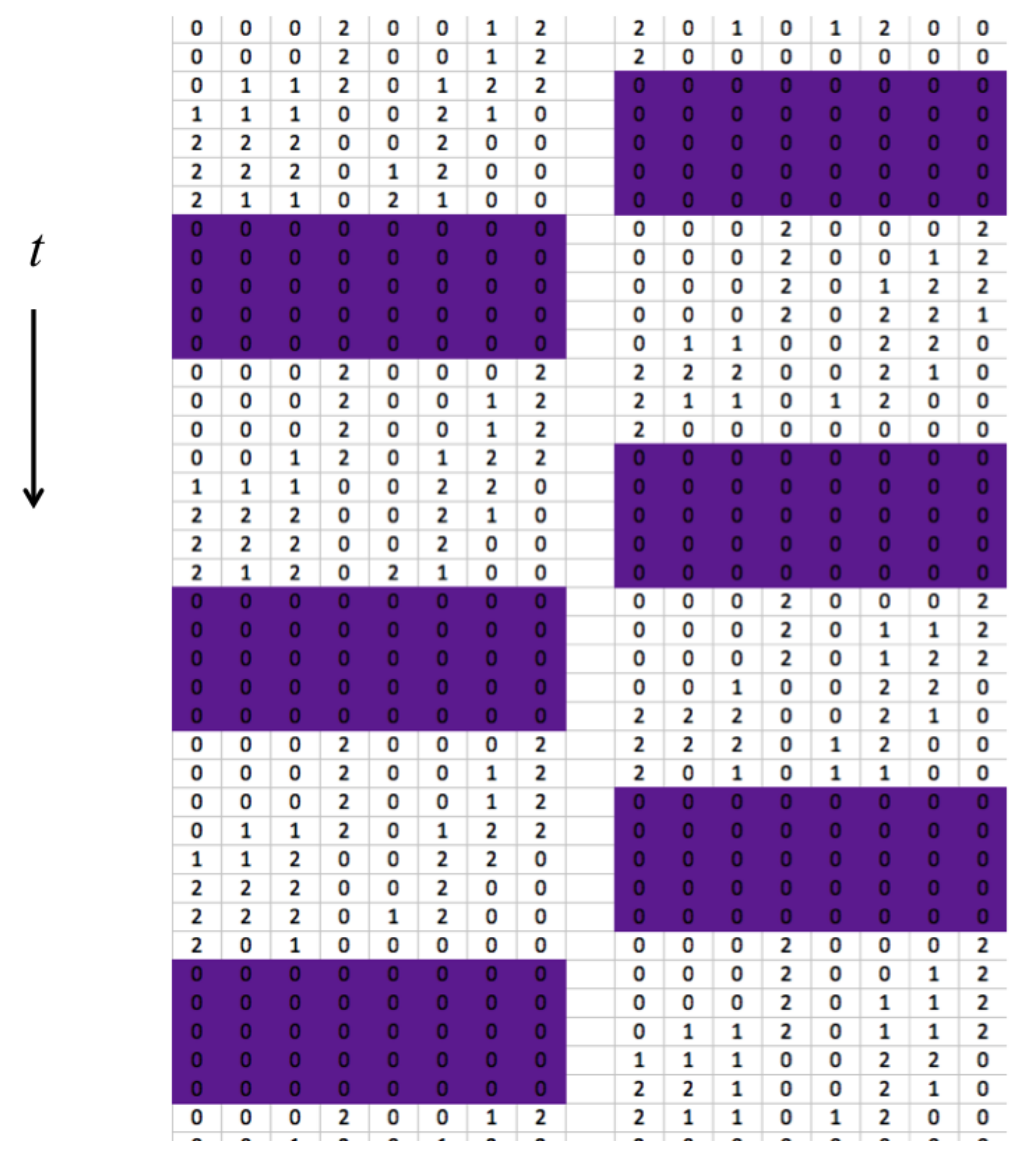


Figure 3. Graph of total pressure from the left foot sole over an interval of 10 seconds obtained from the foot monitoring system from the same data as presented in Figure 2. The two dashed lines indicate the upper and lower thresholds used to calculate the step frequency.

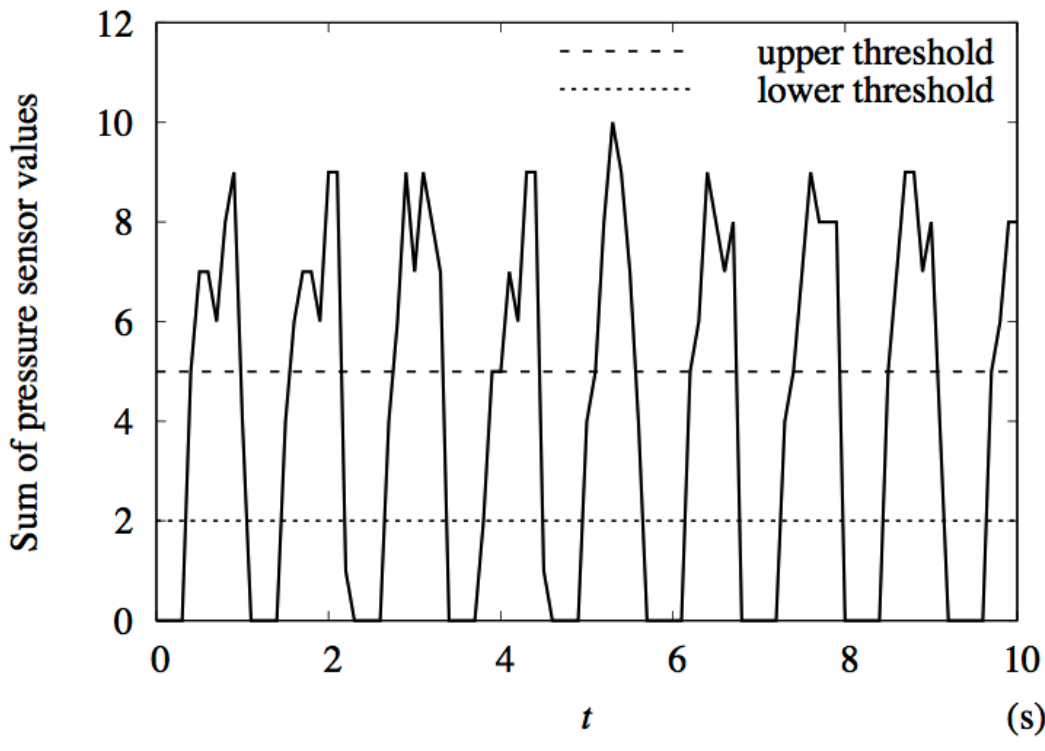

After assigning a status to each time step, we counted the number of transitions from the on-ground to off-ground status and divided it by the time interval to obtain the frequency. As with the gas analyzer data, we omitted data for the first $50 \mathrm{~s}$ and the final $10 \mathrm{~s}$. Only one shoe insole is required to calculate the step frequency; however, we used the average of both sides in this study.

\section{Model}

Our model was constructed from considering the energy changes involved in walking. Suppose a participant with body mass $M u$ is walking with average speed $N u$ on a surface inclined by Theta from the horizontal. The participant is swinging their legs with frequency $f$. The energy consumption rate, Rho, is given by equation 1 (Figure 4). Here positive and negative values of the slope, Theta, of the walking surface correspond to walking uphill and downhill, respectively. $R h o_{\mathrm{K}}$ and $R h o_{\mathrm{U}}$ are rates of changes in the kinetic energy and in the potential energy, respectively, whereas coefficients Gamma, $b_{\text {Tau }}, b_{1}$, and $R h o_{0}$ are parameters to be determined empirically from the data. The energy change rates for $R h o_{\mathrm{K}}$ and $R h o_{\mathrm{U}}$ are given in equation 2 (Figure 4). In the following, we give an explanation of each term in consideration of energy.

\section{Kinetic Energy Component}

We first consider walking on a horizontal surface (ie, Theta $=0$ ). When walking on a treadmill, the upper body moves in a relatively constant velocity, with the moving legs supporting this movement. The legs swing back and forth relative to the upper body's position, undergoing an acceleration-deceleration cycle. We postulated that the energy expenditure was proportional to the kinetic energy change of the legs. The work done on the legs during each walking cycle is given by equation 3 (Figure 4). Here $m$ is the mass of each leg, $v_{0}$ is the maximum

speed of each leg's center of mass, and the factor of 4 accounts for the two legs each undergoing acceleration and then deceleration. This differs from the assumption that the legs swing like a pendulum, in which case gravity would do the work.

Since we usually have no way to easily measure leg mass or leg velocity, we defined two ratios: (1) the ratio $\alpha$ of the leg mass, $m$, to the body mass, $M$; and (2) the ratio $\beta$ of the maximum velocity, $v_{0}$, of the leg to the average walking speed, $v$ (equation 4 in Figure 4).

This allowed us to rewrite equation 3 as $W=2 \alpha \beta M^{2} v^{2}$, giving an expression for the work done per cycle. Assuming that the human body converts chemical energy into kinetic energy with efficiency $\eta_{K}$, the energy consumption rate due to the kinetic energy is given by equation 5 (Figure 4). In writing the right-hand side of equation 5 , the measurable terms are grouped into $P_{K}$ as in equation 2 , whereas the rest are grouped into dimensionless coefficient $\gamma$, given by equation 6 .

\section{Potential Energy Component}

When walking on a horizontal surface perpendicular to the direction of gravity, there is no net change in potential energy. It changes when the subject is walking up or down a slope. We first considered upward inclines. When one walks up a slope of angle Theta at speed $v$ parallel to the surface, their potential energy, $U$, changes at a rate $d U / d t=P_{U}$, given by equation 2 (Figure 4). For simplicity, we further assumed that when walking up a slope, additional energy proportional to this term is required. Accordingly, the energy expenditure rate associated with the changing potential energy is given by $b_{0} P_{U}$, where $b_{0}$ is the inverse of the efficiency, $\eta_{U}$, (equation 7 in Figure 4) with which the body converts stored energy to potential energy. 
Figure 4. List of equations of the model of energy expenditure during walking.

$$
\begin{aligned}
& P=\left\{\begin{array}{lr}
\gamma P_{K}+b_{0} P_{U}+P_{0} & \text { if } \theta \geq 0 \\
\gamma P_{K}+b_{0} P_{U}+b_{1} P_{0}^{-1} P_{U}^{2}+P_{0} & \text { if } \theta<0
\end{array}\right. \\
& P_{K}=2 M v^{2} f, \\
& P_{U}=M g v \sin \theta \\
& W=4 \times \frac{1}{2} m v_{0}^{2} \\
& \alpha \equiv \frac{m}{M}, \quad \beta \equiv \frac{v_{0}}{v} \\
& \frac{1}{\eta_{K}} W f=2 \gamma M v^{2} f=\gamma P_{K} \\
& \gamma \equiv \frac{\alpha \beta^{2}}{\eta_{K}} \\
& b_{0}=\frac{1}{\eta_{U}} \\
& b_{0} P_{U}+b_{1} P_{0}^{-1} P_{U}^{2} \\
& \left|P-P^{\prime}\right| / P^{\prime} \\
& v \sim f h
\end{aligned}
$$

One might consider simply using the same formula for downhill inclines, in which case the term $b_{0} P_{U}=b_{0} M g v \sin$ Theta becomes negative. This would imply that when walking downslope, the change in potential energy can be converted into kinetic energy, thereby subtracting from the total energy cost. However, this leads to a nonsensical result for higher slopes, as it can lead to negative energy consumption. When a downhill slope is steeper than a certain angle, the subject would need to exert a frictional force to prevent from falling forward or walking too fast. Therefore, $b_{0} P_{U}$ does not provide an adequate description of the energy expenditure in this case.
Figure 5 and 6 present scatterplots of the data in the three-dimensional space $\left(P_{K}, P_{U}, P\right)$ for women and men, respectively. This visualization shows that $P$ first decreases then increases as $P_{U}$ is decreased from zero. Such a parabolic shape indicates the presence of a quadratic term; thus, we added to $P$ a term proportional to $P_{U}^{2}$. The energy expenditure associated with potential energy in the case of downhill walking is given by equation 8 (Figure 4). The second term is multiplied by $P_{0}^{-1}$ so that the coefficient $b_{1}$ is kept dimensionless. In other words, $b_{1}$ is the coefficient of the quadratic term in the case of downhill walking in units of $P_{0}$. This leads to the full model, described by equation 1 (Figure 4 ). 
Figure 5. Three-dimensional scatterplot of data (dots) and model prediction (lines) of $P$ versus $P_{U}$ and $P_{K}$ for women.

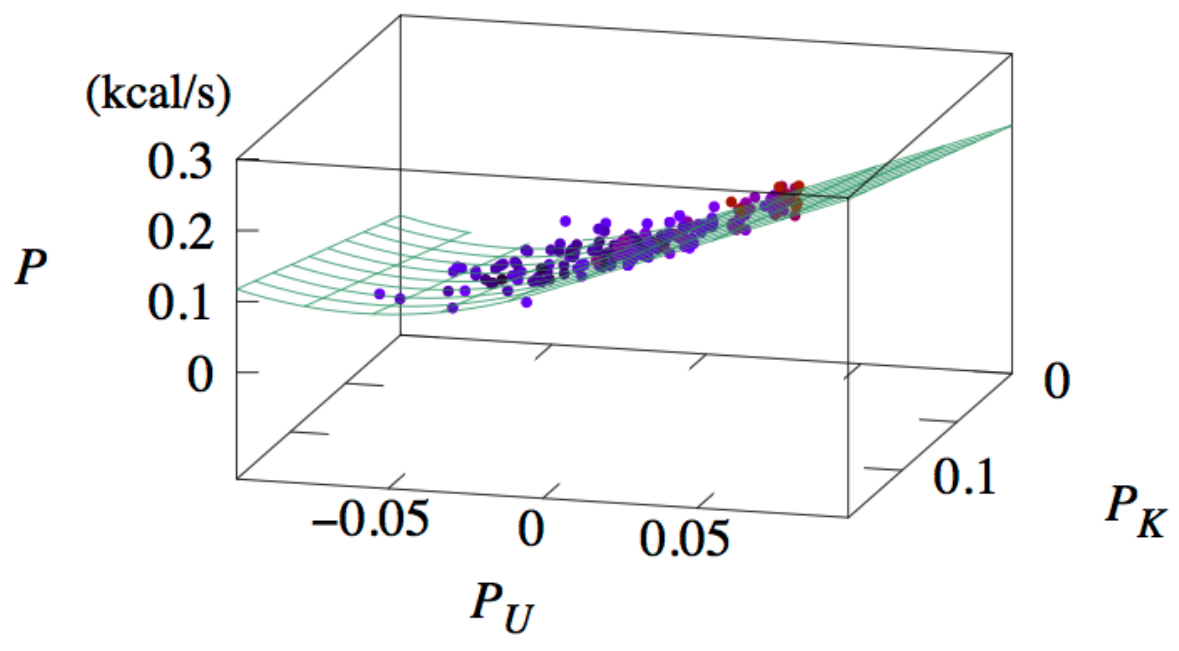

Figure 6. Three-dimensional scatterplot of data (dots) and model prediction (lines) of P versus PU and PK for men.

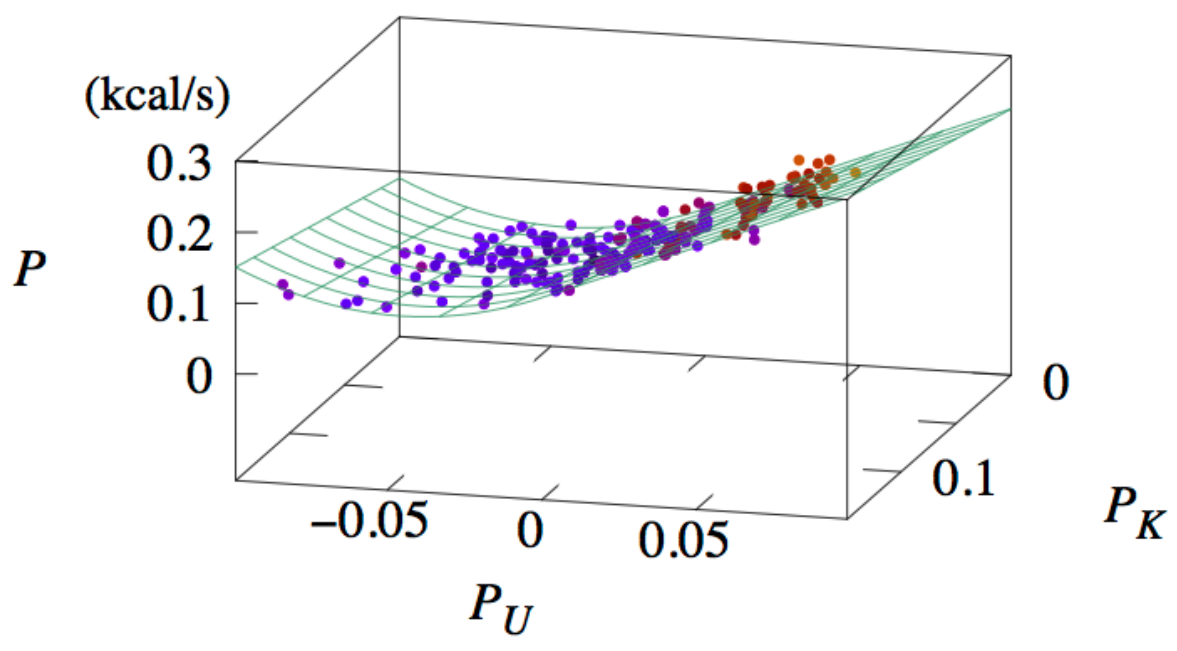

\section{Linear Regression}

The preceding model described leaves parameters $\Upsilon, b_{0}, b_{1}$, and $P_{0}$ to be determined. We obtained these parameters by first taking data for flat and uphill surfaces (Theta $\geq 0)$ and performing multiple linear regression through the use of the first equation in equation 1 (Figure 4) with $\mathrm{Y}, b_{0}$, and $P_{0}$ as fitting parameters. The adjusted $R^{2}$ value for the fits of both women and men was .83. Then $b_{1}$ was obtained via fitting the second equation of equation 1 (Figure 4) to flat and downslope data (Theta $\leq 0)$.
During this secondary fit, $\Upsilon, b_{0}$, and $P_{0}$ were set constant at the values obtained earlier.

\section{Results}

The full set of coefficients, obtained through linear regression, is given in Table 1. The dependency of $P$ on $P_{K}$ and $P_{U}$ is represented by the surfaces in Figures 5 and 6 . Due to the piecewise functional form of the model (equation 1 in Figure 4), the prediction plane has no curvature for $P_{U}>0$ but does in the region $P_{U}<0$.

Table 1. Coefficients for the full model reported with the root-mean-square deviation (RMSD) on comparison with data. The values were obtained by two linear regressions.

\begin{tabular}{llll}
\hline Coefficient & Units & Women & Men \\
\hline$\gamma$ & - & 0.662 & 0.517 \\
$b_{0}$ & - & 1.591 & 1.694 \\
$b_{1}$ & - & 0.575 & 1.086 \\
$P_{0}$ & $\mathrm{kcal} / \mathrm{s}$ & 0.042 & 0.058 \\
RMSD & $\mathrm{kcal} / \mathrm{s}(\mathrm{kcal} / \mathrm{min})$ & $0.016(0.96)$ & $0.016(0.96)$ \\
\hline
\end{tabular}


The fit resulted in a root-mean-square deviation (RMSD) of $0.96 \mathrm{kcal} / \mathrm{min}$ for both women and men. A boxplot of the percentage errors of all trials is given in Figure 7, in which the errors have been calculated according to equation 9 in Figure 4.

Here $P$ is the prediction by the method whereas $P^{\prime}$ is the standard given by the gas analyzer. The median errors were $16.9 \%$ for women, $11.2 \%$ for men, and $12.4 \%$ for both groups. These errors are substantially lower than those found in a validation study for multiple commercial devices, which yielded median accuracies of $28.6 \%$ to $35.0 \%$ across devices for walking [37].

The predictions made by Fitbit Surge had an RMSD of $2.58 \mathrm{kcal} / \mathrm{min}$ (2.7 times that of the model) and a median percent error of $37.3 \%$ (3 times that of the model). However, this high error was mostly due to inaccuracies in sloped walking. When restricted to flat surfaces, the Fitbit Surge's accuracy increased dramatically, whereas the model's accuracy increased moderately. The Fitbit Surge's RMSD on flat surfaces was $1.82 \mathrm{kcal} / \mathrm{min}$ ( 2.3 times that of the model, $0.79 \mathrm{kcal} / \mathrm{min}$ ), and the median percent error was $18.4 \%$ (1.6 times that of the model,
11.2\%). Distributions of percent errors are portrayed with boxplots in Figure 7.

Before discussing the implications of these results, we note that the variables $v$ and $f$ are not independent. If $l$ is the average length of a step, then $v=f l$. Assuming the approximate relation $h \approx l$, where $h$ is the subject's height, we obtain $v \sim f h$ (equation 10 in Figure 4). This relation was observed in the data, as shown in Figure 8.

Equation 7 implies that $\eta_{U}=0.547$ for women and 0.596 for men. In principle, $\Upsilon$ depends on $\alpha, \beta$, and $\eta_{K}$. We assumed the average value of $\alpha=0.185$ for women and 0.165 for men, obtained from an anatomical reference [42], and that $\eta_{K}=\eta_{U}$. Taking these values and the fitting result for $\Upsilon$, we obtained from equation 6 (Figure 4) the ratio $\beta$ with values 1.47 for women and 1.36 for men. This difference in the average may reflect the difference in the average height between women and men. Specifically, equations 4 and 10 (Figure 4) imply $\beta=v_{0} / v$ $\sim v_{0} / f h$. The ratio of the value of $\beta$ for women to that for men equaled 1.08 , whereas the ratio of the average height of men to that of women equaled 1.11 .

Figure 7. Boxplots of the percent errors of predictions made by the model and Fitbit Surge. Errors have been estimated via equation 9 in Figure 4.

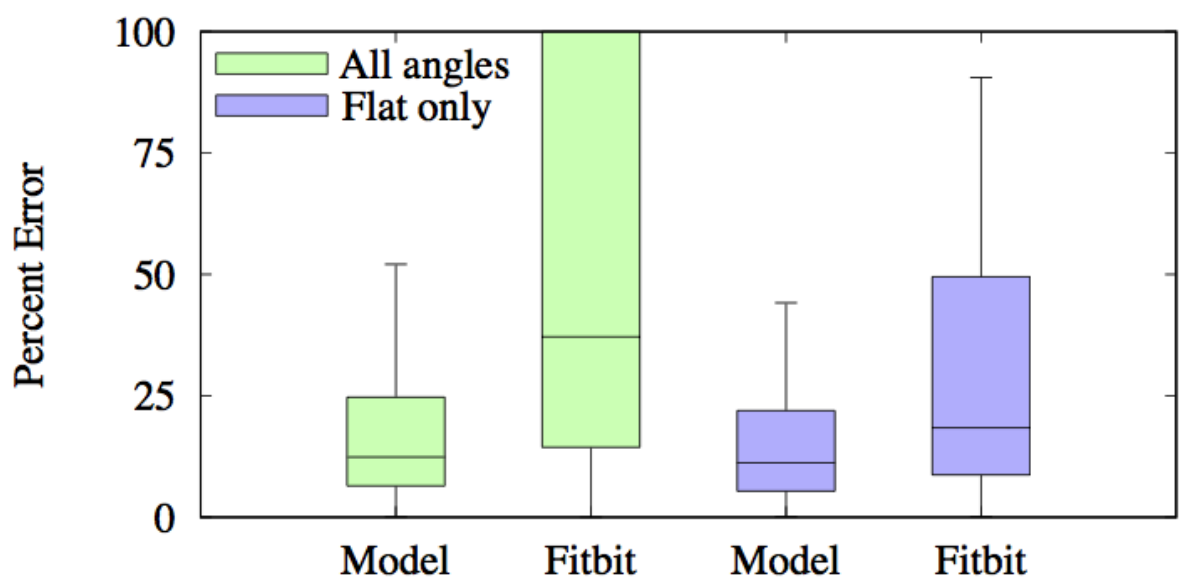

Figure 8. Step frequency, $f$, multiplied by height, $h$, plotted against average walking speed, $v$. Least squares fit line fh $=0.52 \mathrm{v}+1.02(\mathrm{~m} / \mathrm{s})$ is also shown.

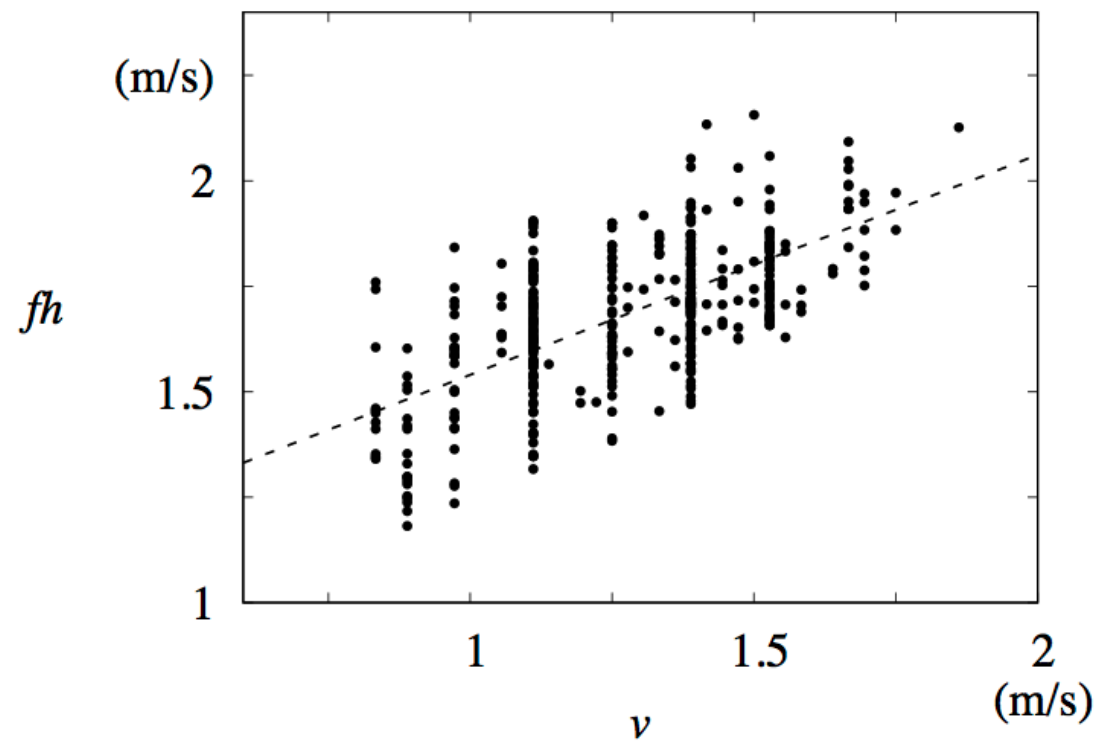




\section{Discussion}

\section{Principal Results}

We developed a model based on rates of change in kinetic and potential energies. In general, it predicts linear dependence of the energy consumption on these rates; in particular, it predicts quadratic dependence of the energy consumption on the potential energy change in the case of downhill walking. The method, used in conjunction with a foot monitoring system, predicts energy expenditure with an RMSD of $0.98 \mathrm{kcal} / \mathrm{min}$ and a median percent error of $12.4 \%$, lower than those of wrist-worn commercial devices in predicting energy expenditure for walking. With one simple piecewise function, the model adequately predicts energy expenditure for walking in a wide range of the gradient.

Notice the differences in parameter values between women and men. The appreciable difference in the value of $b_{1}$ between men and women may result from the difference in walking posture; this is beyond the scope of this work and left for future study. In principle, the parameters are fit for each individual and should vary by subject. Thus, Table 1 presents average values of the coefficients within each gender. Even so, it is remarkable that a high degree of accuracy is observed.

\section{Limitations}

Although the model accounts for varying body mass and step frequency (cadence), this does not account for additional individual variations in parameter values due to walking gait and body dimensions. There may be ways to account for such variations without complicating the model. In addition, because the treadmill incline lies between $14^{\circ}$ uphill and $14^{\circ}$ downhill, we are not able to validate the model for more extreme slopes [43]. In addition, the method has not been tested and calibrated for outdoor walking or variable temperatures and altitudes. However, we believe that our pilot study provides a groundwork for follow-up studies under more ambulatory conditions.

\section{Comparison With Prior Work}

Prior studies have noted the strong correlations between $P$ and $v^{2}$ for level walking [10]. The authors have also similarly considered additional energy expenditure when walking uphill, attributing it to vertical lift work. In contrast, our study proposes a simple formula that predicts energy consumption reasonably well for horizontal, uphill, and downhill surfaces within a unified framework. In addition, Cotes and Meade [9] made use of individual measurements, including resting metabolic rate and leg length. Our model shows that high accuracy can be achieved via reasonable assumptions used in conjunction with a wearable, mobile device.

Other existing studies have studied energy expenditure during uphill and downhill walking $[43,44]$. The authors reported a minimum energy cost when walking $10^{\circ}$ downhill, which is consistent with our results. These studies did not incorporate varying walking speed and body weight, and relied on regression analysis with those variables kept constant. Our study offers a simple formula that applies to various walking speeds and subjects, while also accounting for the surface gradient.

Our method fits separately for women and men. Prior validation studies have found differences in the accuracy of devices between the two genders. A comparative validation study found that gender was one of the strongest predictors for accuracy, with a rate significantly higher for men than for women [37]. Our results suggest that similar error rates for both genders can be achieved.

\section{Conclusions}

We have developed a model that predicts energy expenditure during walking on a gradient surface between $14^{\circ}$ uphill and $14^{\circ}$ downhill, with an RMSD of $0.98 \mathrm{kcal} / \mathrm{min}$. The model has been used in conjunction with a wearable device, the foot monitoring system, which directly measures footsteps. Thus, it offers an accessible method of measuring energy expenditure in realistic walking settings, where gradient walking is common. Future work may test equation 1 (Figure 4) in a wider range of values in the $P_{K}-P_{U}$ space. Testing the method on outdoor walking is also desirable for further validation. Although not yet explored, the device could also be used in conjunction with other activity monitoring devices, such as wrist-worn ones, to produce more accurate measures of energy expenditure.

\section{Acknowledgments}

MY Choi acknowledges support from National Research Foundation of Korea through the Basic Science Research Program (grant No. 2016R1D1A1A09917318 and 2019 R1F1A1046285). MJ Shin and J-J Park acknowledge support from the Wearable Device R\&D Project, Pusan National University Hospital, and KT (grant CMITKT-05).

\section{Conflicts of Interest}

None declared.

\section{References}

1. Nocon M, Hiemann T, Müller-Riemenschneider F, Thalau F, Roll S, Willich SN. Association of physical activity with all-cause and cardiovascular mortality: a systematic review and meta-analysis. Eur J Cardiovasc Prev Rehabil 2008 Jun;15(3):239-246. [doi: 10.1097/HJR.0b013e3282f55e09] [Medline: 18525377]

2. Wen CP, Wai JP, Tsai MK, Yang YC, Cheng TY, Lee M, et al. Minimum amount of physical activity for reduced mortality and extended life expectancy: a prospective cohort study. Lancet 2011 Oct 1;378(9798):1244-1253. [doi:

10.1016/S0140-6736(11)60749-6] [Medline: 21846575] 
3. Trost SG, Blair SN, Khan KM. Physical inactivity remains the greatest public health problem of the 21st century: evidence, improved methods and solutions using the '7 investments that work' as a framework. Br J Sports Med 2014 Jan 10;48(3):169-170. [doi: 10.1136/bjsports-2013-093372]

4. Maddison R, Gemming L, Monedero J, Bolger L, Belton S, Issartel J, et al. Quantifying human movement using the Movn smartphone app: validation and field study. JMIR Mhealth Uhealth 2017 Aug 17;5(8):e122. [doi: 10.2196/mhealth.7167] [Medline: 28818819]

5. Montoye HJ, Washburn R, Servais S, Ertl A, Webster JG, Nagle FJ. Estimation of energy expenditure by a portable accelerometer. Med Sci Sports Exerc 1983;15(5):403-407. [Medline: 6645869]

6. Meijer GA, Westerterp KR, Koper H, ten Hoor F. Assessment of energy expenditure by recording heart rate and body acceleration. Med Sci Sports Exerc 1989 Jun;21(3):343-347. [Medline: 2733585]

7. Chen KY, Sun M. Improving energy expenditure estimation by using a triaxial accelerometer. J Appl Physiol (1985) 1997 Dec;83(6):2112-2122 [FREE Full text] [doi: 10.1152/jappl.1997.83.6.2112] [Medline: 9390989]

8. Passmore R, Durnin JV. Human energy expenditure. Physiol Rev 1955 Oct 01;35(4):801-840. [doi: 10.1152/physrev.1955.35.4.801]

9. Cotes JE, Meade F. The energy expenditure and mechanical energy demand in walking. Ergonomics 1960 Apr;3(2):97-119. [doi: 10.1080/00140136008930473]

10. Dill DB. Oxygen used in horizontal and grade walking and running on the treadmill. J Appl Physiol 1965 Jan;20(1):19-22. [doi: 10.1152/jappl.1965.20.1.19] [Medline: 14257553]

11. Menier DR, Pugh LG. The relation of oxygen intake and velocity of walking and running, in competition walkers. J Physiol 1968 Aug;197(3):717-721. [doi: 10.1113/jphysiol.1968.sp008584] [Medline: 5666183]

12. van der Walt WH, Wyndham $\mathrm{CH}$. An equation for prediction of energy expenditure of walking and running. J Appl Physiol 1973 May;34(5):559-563. [doi: 10.1152/jappl.1973.34.5.559]

13. Holt KG, Hamill J, Andres RO. Predicting the minimal energy costs of human walking. Med Sci Sports Exerc 1991 Apr;23(4):491-498. [Medline: 1905381]

14. Margaria R, Cerretelli P, Aghemo P, Sassi G. Energy cost of running. J Appl Physiol 1963 Mar 01;18(2):367-370. [doi: 10.1152/jappl.1963.18.2.367]

15. Zarrugh MY, Radcliffe CW. Predicting metabolic cost of level walking. Europ J Appl Physiol 1978;38(3):215-223. [doi: 10.1007/bf00430080]

16. Cavanagh PR, Williams KR. The effect of stride length variation on oxygen uptake during distance running. Med Sci Sports Exerc 1982;14(1):30-35. [doi: 10.1249/00005768-198201000-00006] [Medline: 7070254]

17. Steudel K. Limb morphology, bipedal gait, and the energetics of hominid locomotion. Am J Phys Anthropol 1996;99(2):345-355. [doi: 10.1002/(sici)1096-8644(199602)99:2<345::aid-ajpa9>3.3.co;2-m]

18. Minetti A, Alexander R. A theory of metabolic costs for bipedal gaits. J Theor Biol 1997 Jun;186(4):467-476. [doi: 10.1006/jtbi.1997.0407]

19. Alexander RM. A model of bipedal locomotion on compliant legs. Philos Trans R Soc Lond B Biol Sci 1992 Oct 29;338(1284):189-198. [doi: 10.1098/rstb.1992.0138] [Medline: 1360684]

20. Doke J, Donelan JM, Kuo AD. Mechanics and energetics of swinging the human leg. J Exp Biol 2005 Feb;208(Pt 3):439-445 [FREE Full text] [doi: 10.1242/jeb.01408] [Medline: 15671332]

21. Kuo A. Energetics of actively powered locomotion using the simplest walking model. J Biomech Eng 2002;124(1):113-120. [doi: $10.1115 / 1.1427703]$

22. Kuo AD, Donelan JM, Ruina A. Energetic consequences of walking like an inverted pendulum: step-to-step transitions. Exerc Sport Sci Rev 2005 Apr;33(2):88-97. [Medline: 15821430$]$

23. Ruina A, Bertram JE, Srinivasan M. A collisional model of the energetic cost of support work qualitatively explains leg sequencing in walking and galloping, pseudo-elastic leg behavior in running and the walk-to-run transition. J Theor Biol 2005 Nov;237(2):170-192. [doi: 10.1016/j.jtbi.2005.04.004]

24. Osaki Y, Kunin M, Cohen B, Raphan T. Three-dimensional kinematics and dynamics of the foot during walking: a model of central control mechanisms. Exp Brain Res 2006 Aug 18;176(3):476-496. [doi: 10.1007/s00221-006-0633-1]

25. Donker SF, Mulder T, Nienhuis B, Duysens J. Adaptations in arm movements for added mass to wrist or ankle during walking. Exp Brain Res 2002 Jun 21;146(1):26-31. [doi: 10.1007/s00221-002-1145-2]

26. Zehr EP, Haridas C. Modulation of cutaneous reflexes in arm muscles during walking: further evidence of similar control mechanisms for rhythmic human arm and leg movements. Exp Brain Res 2003 Feb 6;149(2):260-266. [doi: 10.1007/s00221-003-1377-9]

27. Hirasaki E, Moore ST, Raphan T, Cohen B. Effects of walking velocity on vertical head and body movements during locomotion. Exp Brain Res 1999 Jul;127(2):117-130. [doi: 10.1007/s002210050781]

28. Hausdorff JM, Yogev G, Springer S, Simon ES, Giladi N. Walking is more like catching than tapping: gait in the elderly as a complex cognitive task. Exp Brain Res 2005 Apr 28;164(4):541-548. [doi: 10.1007/s00221-005-2280-3]

29. Blin O, Ferrandez A, Serratrice G. Quantitative analysis of gait in Parkinson patients: increased variability of stride length. J Neurol Sci 1990 Aug;98(1):91-97. [doi: 10.1016/0022-510x(90)90184-o] [Medline: 2230833] 
30. Collins SH, Wisse M, Ruina A. A Three-Dimensional Passive-Dynamic Walking Robot with Two Legs and Knees. Int J Robotics Res 2016 Jul 02;20(7):607-615. [doi: 10.1177/02783640122067561]

31. Adam Noah J, Spierer DK, Gu J, Bronner S. Comparison of steps and energy expenditure assessment in adults of Fitbit Tracker and Ultra to the Actical and indirect calorimetry. J Med Eng Tech 2013 Sep 05;37(7):456-462. [doi: 10.3109/03091902.2013.831135]

32. Brugniaux JV, Niva A, Pulkkinen I, Laukkanen RM, Richalet J, Pichon AP. Polar Activity Watch 200: a new device to accurately assess energy expenditure. Br J Sports Med 2008 Apr 15;44(4):245-249. [doi: 10.1136/bjsm.2007.045575]

33. Härtel S, Gnam J, Löffler S, Bös K. Estimation of energy expenditure using accelerometers and activity-based energy models_validation of a new device. Eur Rev Aging Phys Act 2010 Dec 2;8(2):109-114. [doi: 10.1007/s11556-010-0074-5]

34. Takacs J, Pollock CL, Guenther JR, Bahar M, Napier C, Hunt MA. Validation of the Fitbit One activity monitor device during treadmill walking. J Sci Med Sport 2014 Sep;17(5):496-500. [doi: 10.1016/j.jsams.2013.10.241]

35. Diaz KM, Krupka DJ, Chang MJ, Peacock J, Ma Y, Goldsmith J, et al. Fitbit@: An accurate and reliable device for wireless physical activity tracking. Int J Cariol 2015 Apr;185:138-140. [doi: 10.1016/j.ijcard.2015.03.038]

36. Tucker WJ, Bhammar DM, Sawyer BJ, Buman MP, Gaesser GA. Validity and reliability of Nike + Fuelband for estimating physical activity energy expenditure. BMC Sports Sci Med Rehabil 2015 Jun 30;7(1):14. [doi: 10.1186/s13102-015-0008-7]

37. Shcherbina A, Mattsson C, Waggott D, Salisbury H, Christle J, Hastie T, et al. Accuracy in Wrist-Worn, Sensor-Based Measurements of Heart Rate and Energy Expenditure in a Diverse Cohort. J Pers Med 2017 May 24;7(2):3 [FREE Full text] [doi: 10.3390/jpm7020003] [Medline: 28538708]

38. Ainslie PN, Reilly T, Westerterp KR. Estimating human energy expenditure. Sports Med 2003;33(9):683-698. [doi: 10.2165/00007256-200333090-00004]

39. Levine JA. Measurement of energy expenditure. Public Health Nutr 2007 Jan 02;8(7a):1123-1132. [doi: 10.1079/phn2005800]

40. Haugen HA, Chan L, Li F. Indirect calorimetry: a practical guide for clinicians. Nutr Clin Pract 2017 Jan 06;22(4):377-388. [doi: 10.1177/0115426507022004377]

41. Mifflin MD, St JS, Hill LA, Scott BJ, Daugherty SA, Koh YO. A new predictive equation for resting energy expenditure in healthy individuals. Am J Clin Nutr 1990 Feb 01;51(2):241-247. [doi: 10.1093/ajcn/51.2.241]

42. Plagenhoef S, Evans FG, Abdelnour T. Anatomical data for analyzing human motion. Res Q Exerc Sport 1983 Jun;54(2):169-178. [doi: 10.1080/02701367.1983.10605290]

43. Minetti AE, Moia C, Roi GS, Susta D, Ferretti G. Energy cost of walking and running at extreme uphill and downhill slopes. J Appl Physiol 2002 Sep 01;93(3):1039-1046. [doi: 10.1152/japplphysiol.01177.2001]

44. Minetti AE, Ardigo LP, Saibene F. Mechanical determinants of gradient walking energetics in man. J Physiol 1993 Dec 01;471(1):725-735. [doi: 10.1113/jphysiol.1993.sp019969]

\title{
Abbreviations \\ RMSD: root-mean-square deviation
}

\author{
Edited by G Eysenbach; submitted 27.09.18; peer-reviewed by M Stuckey, T Ebara, B Chaudhry, J Seitz; comments to author 01.04.19; \\ revised version received 10.05.19; accepted 19.07.19; published 23.10.19 \\ Please cite as: \\ Kim SH, Kim JW, Park JJ, Shin MJ, Choi M \\ Predicting Energy Expenditure During Gradient Walking With a Foot Monitoring Device: Model-Based Approach \\ JMIR Mhealth Uhealth 2019;7(10):e12335 \\ URL: https://mhealth.jmir.org/2019/10/e12335 \\ doi: $\underline{10.2196 / 12335}$ \\ PMID: 31647467
}

(CSoon Ho Kim, Jong Won Kim, Jung-Jun Park, Myung Jun Shin, MooYoung Choi. Originally published in JMIR Mhealth and Uhealth (http://mhealth.jmir.org), 23.10.2019. This is an open-access article distributed under the terms of the Creative Commons Attribution License (https://creativecommons.org/licenses/by/4.0/), which permits unrestricted use, distribution, and reproduction in any medium, provided the original work, first published in JMIR mhealth and uhealth, is properly cited. The complete bibliographic information, a link to the original publication on http://mhealth.jmir.org/, as well as this copyright and license information must be included. 